\title{
Abdominal wall and labial edema presenting in a girl with Henoch-Schönlein purpura: a case report
}

\author{
Rania Hiram-Karasmanis ${ }^{1}$, Ronald Garth Smith ${ }^{1 *}$, Maria Radina² ${ }^{2}$ Donald Allen Soboleski ${ }^{3}$
}

\begin{abstract}
Introduction: Henoch-Schönlein purpura is a common immunoglobulin A-mediated vasculitic syndrome in children, characterized by purpuric rash, arthritis and abdominal pain. Renal involvement, manifested by the presence of hematuria and/or proteinuria, is also frequently seen. In most cases, patients with this disease achieve complete recovery, but some progress to renal impairment. Gastro-intestinal manifestations are present in twothirds of affected patients and range from vomiting, diarrhea, and peri-umbilical pain to serious complications such as intussusception and gastrointestinal hemorrhage.
\end{abstract}

Case presentation: We report the case of a 7-year-old Caucasian girl who presented with abdominal pain, labial swelling, and a large abdominal ecchymosis two weeks after having been diagnosed with Henoch-Schönlein purpura. A computed tomography scan revealed abdominal wall edema extending to the groin, without any intraabdominal pathology. She was successfully treated with intravenous steroids.

Conclusion: Circumferential anterior abdominal wall edema and labial edema have never been reported previously, to the best of our knowledge, as a complication of Henoch-Schönlein purpura. These findings further contribute to the wide spectrum of manifestations of this disorder in the literature, aiding in its recognition and management.

\section{Introduction}

Henoch-Schönlein purpura (HSP) is an IgA-mediated vasculitis that presents with the common tetrad of abdominal pain, arthritis, purpuric rash and renal involvement. It is usually a benign disease of childhood, typically affecting children between the ages of four and seven years, who achieve complete recovery in most cases. HSP is often preceded by an upper respiratory tract infection, with Group A beta-hemolytic streptococcus responsible for up to $50 \%$ of the occurrences [1].

The diagnostic criteria of the American College of Rheumatology for HSP are the following: palpable purpura; patient is aged 20 years or above; acute abdominal pain; and biopsy showed granulocytes in the walls of small arterioles or venules [2].

The presence of two or more of these criteria has a sensitivity and specificity of $87.1 \%$ and $87.7 \%$, respectively, for the diagnosis of HSP. A subsequent review of the classification of childhood vasculitides was carried out in 2005 and the diagnostic criteria modified

\footnotetext{
* Correspondence: gs3@queensu.ca

'Department of Pediatrics, Queen's University, Kingston, ON, Canada
}

(EULAR/PReS consensus report, 2006) [3]. These new criteria include:

Palpable purpura (mandatory criterion) in the presence of at least one of the following four features: Diffuse abdominal pain; any biopsy showing predominant IgA deposition; arthritis (acute, any joint) or arthralgia; renal involvement (any hematuria and/or proteinuria).

Gastrointestinal manifestations seen with HSP have been well described, and vary in their severity. In a study of 100 patients with HSP, Saulsbury reported that $63 \%$ of patients complained of abdominal pain [1] with typical symptoms including colicky abdominal pain, vomiting and gastrointestinal bleeding. Gastro-intestinal symptoms are the result of extravasation of blood and fluid into the bowel wall, leading to ulceration of the bowel mucosa and, eventually, bleeding, commonly involving the jejunum and ileum [4]. Intussusception is a rare but serious complication of HSP, occurring in 1-5\% of patients [4]. In the past few years, there have been several case reports of new gastro-intestinal manifestations of HSP, including hemorrhagic ascites [5], perforation of large and small bowel [6], pancreatitis [7] and ischemic necrosis of the bile ducts [8]. 
Ultrasound is typically the modality of choice for investigation of abdominal pain associated with HSP, and can detect mural thickness and hematoma, ileus, peritoneal fluid and intussusception [9].

The treatment of HSP remains mainly supportive, as the acute symptoms resolve spontaneously in the majority of patients. For more severe cases with serious complications of the disease, treatment commonly includes corticosteroids, immunosuppressive drugs, and plasmapheresis [10]. The use of corticosteroids in the treatment of HSP remains anecdotal, as no prospective placebo controlled trials have been done [1]. A recent systematic review has been carried out by Weiss et al. in 2007 [11].

\section{Case presentation}

A previously healthy 7-year-old Caucasian girl was admitted to our hospital with a history of abdominal pain, labial swelling and a large ecchymosis extending from the left subcostal area to the left lower quadrant. Two weeks before being admitted to the hospital, she experienced symptoms of an upper respiratory infection followed by joint discomfort, peripheral edema and a palpable, purpuric rash. She presented to a smaller community hospital, where the additional findings of hypertension and a Group A beta-hemolytic streptococcus-positive throat swab were discovered by a consulting pediatrician. She was diagnosed with Henoch-Schönlein purpura (HSP) and treated with two weeks of Penicillin
$\mathrm{V}$ for her tonsillitis. Her symptoms improved, but over the course of the next six days, she developed increasing abdominal pain and "distention". She also experienced significant pain in her genital area with associated labial swelling. She was transferred to our institution, a tertiary care Pediatric Center, for further evaluation. There was no history of abdominal trauma.

On admission, she was found to be hypertensive, with a blood pressure just above the 90th percentile for age and height. She had an exudative plaque on the left tonsil, but a throat swab was negative. Her abdomen revealed a large ecchymosis, $10 \mathrm{~cm}$ in diameter over the left quadrant, with significant edema/swelling extending from the left flank to the labia majora (Figure 1). The abdomen was diffusely tender to palpation, and a faint raised papular rash was noted on her lower abdomen, buttocks and lower extremities. A complete blood count revealed a leukocytosis with a left shift and a normal platelet count. Urine analysis was positive for blood (microscopic) and an abdominal ultrasound was "normal" but incomplete. The ultrasound was performed to rule out intussusception, but had to be abandoned due to the extreme abdominal wall tenderness. A computed tomography $(\mathrm{CT})$ scan of the abdomen was then performed, and this revealed markedly increased attenuation within the subcutaneous tissues of the left side of the abdominal wall and flank, extending to the groin and labia majora, consistent with hemorrhage or edema (Figure 2, 3). No intra-abdominal pathology was seen.

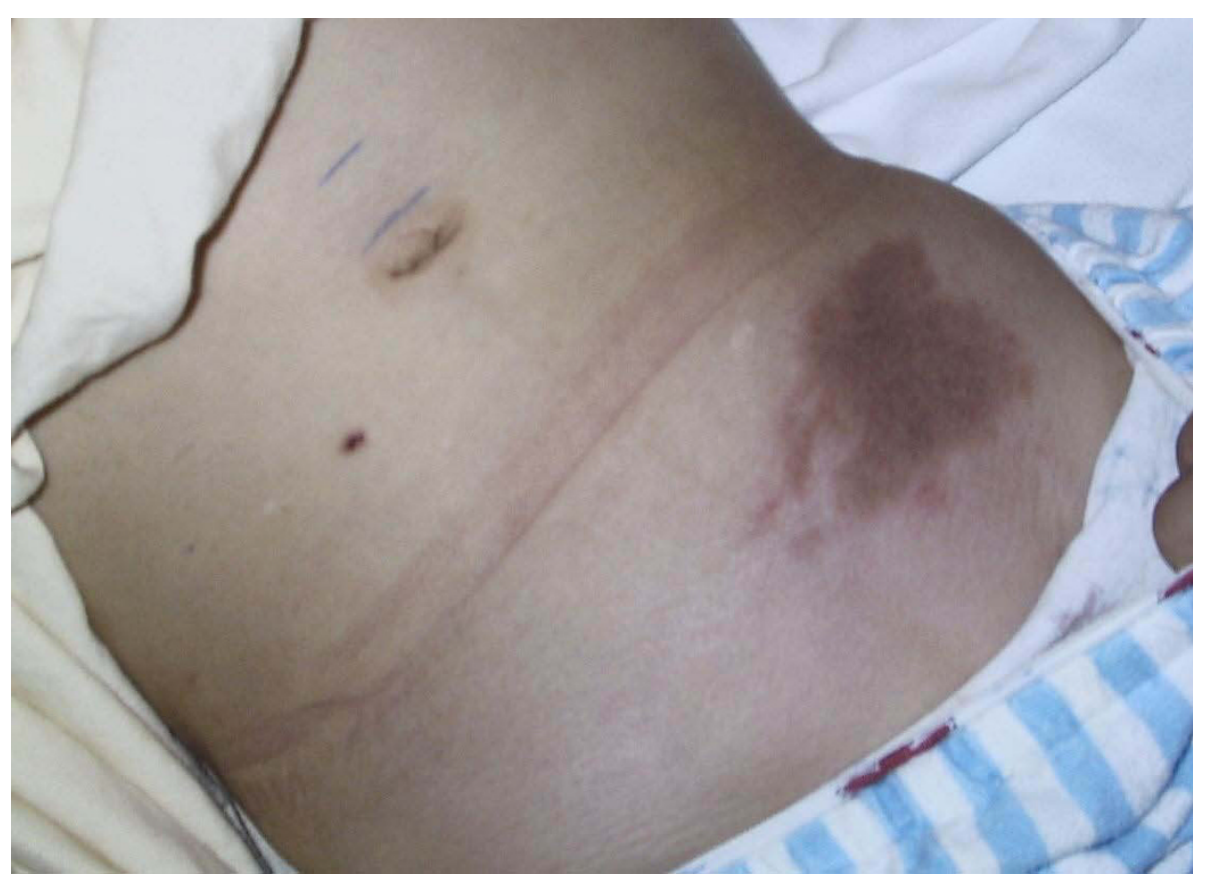

Figure 1 Abdominal ecchymosis. Large ecchymosis over the left lower quadrant and edema of the left flank. 


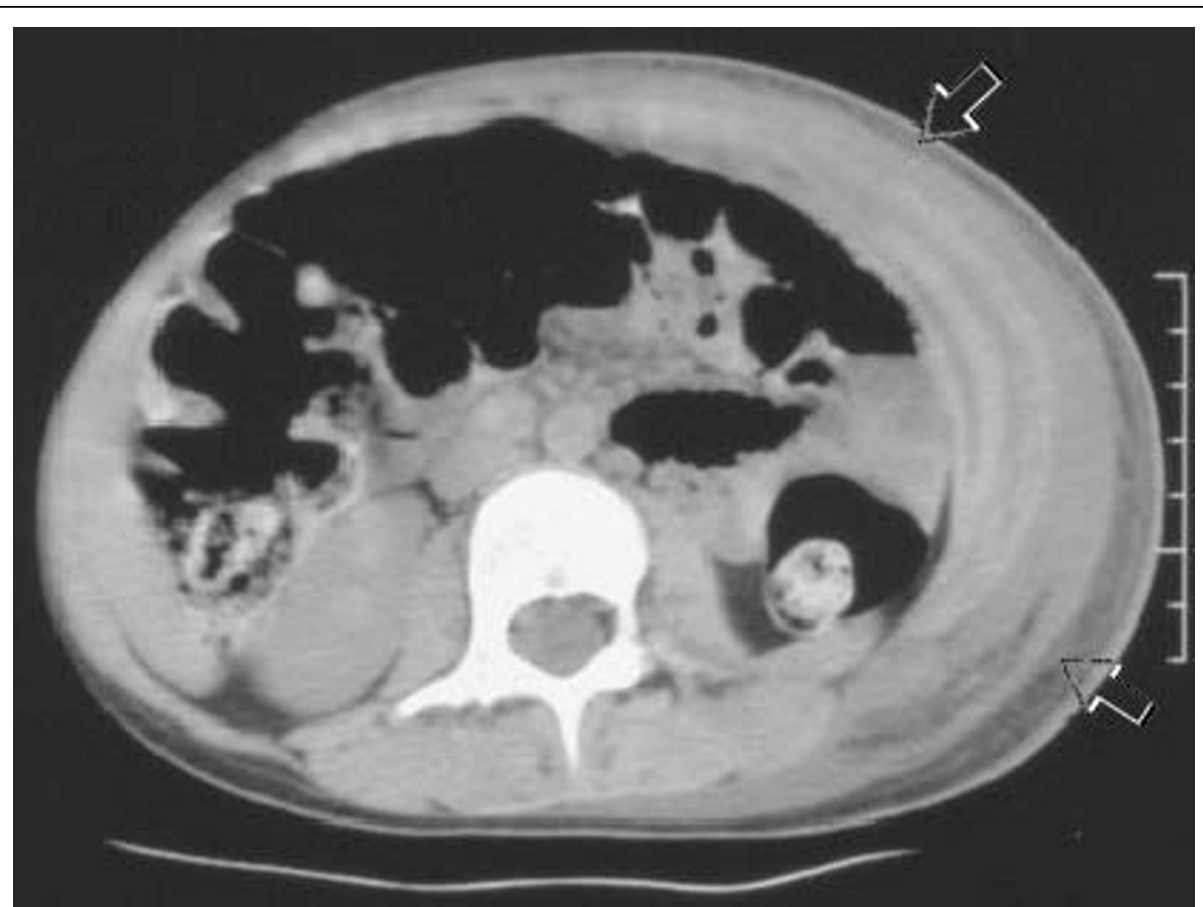

Figure 2 Abdominal wall edema. Unenhanced axial computed tomography scan through the mid-abdomen demonstrates extensive fluid tracking between abdominal wall muscle layers and coursing along anterior margins within subcutaneous fat (arrows).

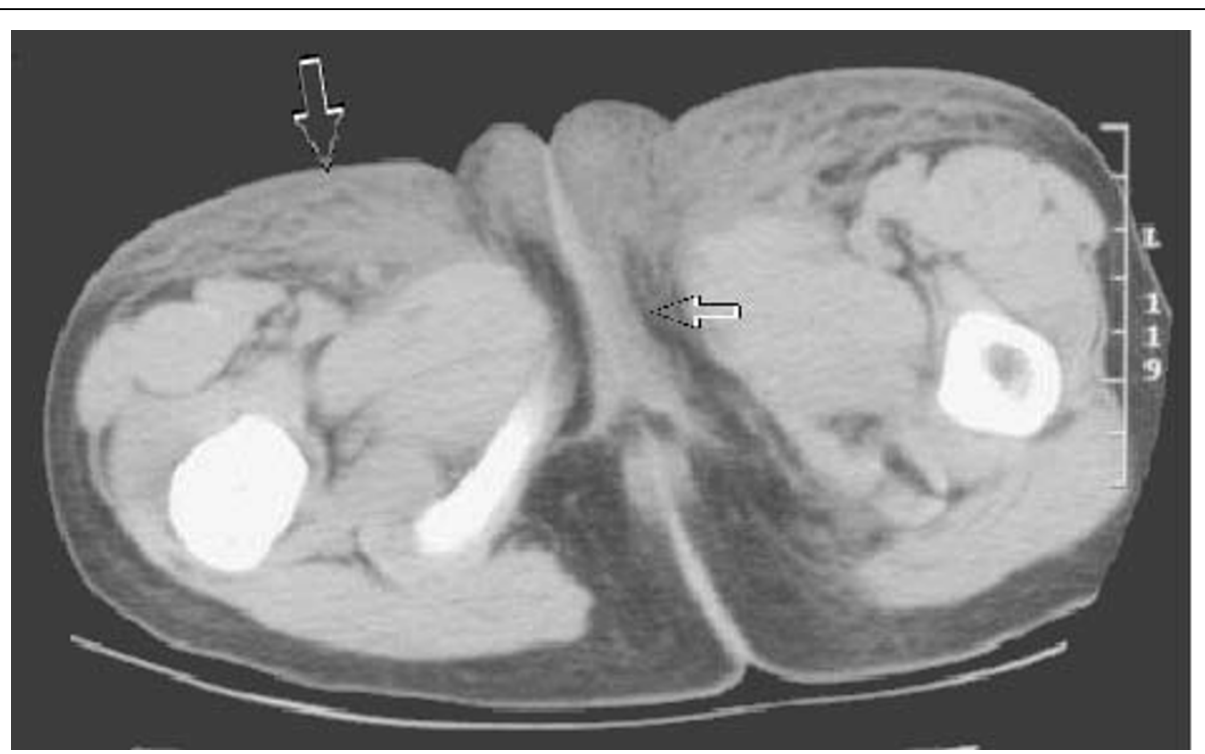

Figure 3 Labial edema. Unenhanced axial computed tomography image depicts extensive bilateral subcutaneous edema (large arrow) with extension into the labia (small arrow).

Our patient was treated with $2 \mathrm{mg} / \mathrm{kg} /$ day of intravenous methylprednisolone for 48 hours, and then switched to $2 \mathrm{mg} / \mathrm{kg} /$ day of oral prednisone for seven days. Within 36 hours of initiation of treatment, her abdominal pain and distention improved significantly and her hematuria resolved.

\section{Discussion}

The reported case describes circumferential anterior abdominal wall edema and labial edema as a complication of HSP. Gastrointestinal manifestations of HSP are common. However, they usually result from edema and ulceration of the bowel wall, leading to pain and 
hemorrhage. Although only a minority of patients with HSP are investigated with a CT scan, findings usually include multifocal bowel wall thickening with skip areas, mesenteric edema, vascular engorgement and non-specific lymphadenopathy [12]. Our patient had a normal bowel wall, but instead had significant circumferential edema within the abdominal wall and the left flank - a finding that has not been previously reported. A CT scan was performed mainly because of the acute tenderness of the abdominal wall, which precluded satisfactory completion of the ultrasound study.

Genital involvement in HSP has been previously documented in males, and consisted of orchitis and scrotal swelling found in nine percent of boys in Saulsbury's study [1]. There were also two recent reports of penile edema associated with HSP $[13,14]$. Aside from one report of cutaneous vulvar lesions associated with HSP, there have been no other descriptions of involvement of the women's genitalia to date [15]. Our patient had clinical and radiological edema of the labia majora, which was likely an extension of the abdominal wall edema. A finding such as this has never been reported before, and may be the female equivalent of scrotal swelling in boys.

Similar to the responses described in several other reports, our patient had significant improvement within hours of initiation of steroid treatment and achieved almost complete resolution of symptoms within three days.

\section{Conclusion}

This case presents an occurrence of abdominal wall and labial edema as a complication associated with HSP that has not been previously reported. The symptoms resolved promptly upon treatment with steroids. These new findings are of interest, as they further contribute to the wide spectrum of manifestations of HSP already described in the literature, aiding in the recognition and management of this disorder.

\section{Consent}

Written informed consent was obtained from the parent of our patient for publication of this case report and any accompanying images. A copy of the written consent is available for review by the Editor-in-Chief of this journal.

\section{Abbreviations}

CT: Computed tomography; HSP: Henoch-Schönlein purpura; kg: Kilograms; mg: Milligrams.

\section{Acknowledgements}

The authors would like to thank Dr. Christopher J. Justinich, a pediatric gastroenterologist, for helping to prepare the case for poster presentation. The authors have no sources of financial support to disclose.

\section{Author details}

${ }^{1}$ Department of Pediatrics, Queen's University, Kingston, ON, Canada. ${ }^{2}$ Faculty of Health Sciences, Queen's University, Kingston, ON, Canada. ${ }^{3}$ Department of Diagnostic Radiology, Kingston General Hospital, Kingston, ON, Canada.

\section{Authors' contributions}

$\mathrm{RH}$ and RGS conceived of the project and participated in its design. MR and $\mathrm{RH}$ helped draft the manuscript. DS assisted with the interpretation of radiological data. All authors read and approved the final manuscript.

\section{Competing interests}

The authors declare that they have no competing interests.

Received: 1 May 2008 Accepted: 29 March 2010

Published: 29 March 2010

\section{References}

1. Saulsbury FT: Henoch-Schönlein purpura in children. Report of 100 patients and review of the literature. Medicine 1999, 78:395-409.

2. Mills JA, Michel BA, Bloch DA, Calabrese LH, Hunder GG, Arend WP, Edworthy SM, Fauci AS, Leavitt RY, Lie JT, et al: The American College of Rheumatology 1990 criteria for the classification of Henoch-Schönlein purpura. Arthritis \& Rheumatism 1990, 33:1114-1121.

3. Ozen S, Ruperto N, Dillon MJ, Bagga A, Barron K, Davin JC, Kawasaki T, Lindsley C, Petty RE, Prieur AM, Ravelli A, Woo P: EULAR/PReS e ndorsed consensus criteria for the classification of childhood vasculitides. Annals of the Rheumatic Diseases 2006, 65:936-941.

4. Bailey M, Chapin W, Licht $H$, Reynolds JC: The effects of vasculitis on the gastrointestinal tract and liver. Gastroenterology Clinics of North America 1998, 27:747-782.

5. Venuta A, Bertolani P, Garetti E, Venturelli C, Predieri B, Muttini ED, Compagni E: Hemorrhagic ascites in a child with Henoch-Schönlein purpura. Journal of Pediatric Gastroenterology \& Nutrition 1999, 29:358-359.

6. Bissonnette R, Dansereau A, D'Amico P, Pateneaude JV, Paradis J: Perforation of large and small bowel in Henoch-Schönlein purpura. International Journal of Dermatology 1997, 36:361-363.

7. Cheung KM, Mok F, Lam P, Chan KH: Pancreatitis associated with HenochSchönlein purpura. Journal of Paediatrics \& Child Health 2001, 37:311-313.

8. Viola $\mathrm{S}$, Meyer M, Fabre M, Tounian $\mathrm{P}$, Goddon R, Dechelotte $\mathrm{P}$, Valayer J, Gruner M, Bernard O: Ischemic necrosis of bile ducts complicating Schönlein-Henoch purpura. Gastroenterology 1999, 117:211-214.

9. Connolly B, O'Halpin D: Sonographic evaluation of the abdomen in Henoch-Schönlein purpura. Clinical Radiology 1994, 49:320-323.

10. Roberts PF, Waller TA, Brinker TM, Riffe IZ, Sayre JW, Bratton RL: HenochSchonlein purpura: a review article. Southern Medical Journal 2007, 100:821-824.

11. Weiss PF, Feinstein JA, Luan X, Burnham JM, Feudtner C: Effects of corticosteroid on Henoch-Schönlein purpura: a systematic review. Pediatrics 2007, 120:1079-1087.

12. Jeong YK, Ha HK, Yoon CH, Gong G, Kim PN, Lee MG, Min YI, Auh YH: Gastrointestinal involvement in Henoch-Schönlein syndrome: CT findings. AJR American Journal of Roentgenology 1997, 168:965-968.

13. Sandell J, Ramanan R, Shah D: Penile involvement in Henoch-Schönlein purpura. Indian Journal of Pediatrics 2002, 69:529-530.

14. Pennesi M, Biasotto E, Saccari A: Schönlein-Henoch purpura involving the penis. Archives of Disease in Childhood 2006, 91:603.

15. Fischer $\mathrm{G}$, Rogers $\mathrm{M}$ : Vulvar disease in children: a clinical audit of 130 cases. Pediatric Dermatology 2000, 17:1-6.

doi:10.1186/1752-1947-4-98

Cite this article as: Hiram-Karasmanis et al:: Abdominal wall and labial edema presenting in a girl with Henoch-Schönlein purpura: a case report. Journal of Medical Case Reports 2010 4:98. 